\title{
Microstructure and Inertial Characteristics of MHD Suspended SWCNTs and MWCNTs Based Maxwell Nanofluid Flow with Bio-Convection and Entropy Generation Past a Permeable Vertical Cone
}

\author{
Zahir Shah ${ }^{1, *(\mathbb{C}}$, Ebraheem Alzahrani ${ }^{2}{ }^{\mathbb{D}}$, Muhammad Jawad $^{3}$ and Umair Khan ${ }^{4}$ \\ 1 Department of Mathematics, University of Lakki Marwat, Lakki Marwat 28420, Pakistan \\ 2 Department of Mathematics, Faculty of Science, King Abdulaziz University, P. O. Box 80203, \\ Jeddah 21589, Saudi Arabia; eoalzahrani@kau.edu.sa \\ 3 Department of Mathematics, Abdul Wali Khan University, Mardan 23200, Pakistan; \\ muhammadjawad175@yahoo.com \\ 4 Department of Mathematics and Social Sciences, Sukkur IBA University, Sukkur 65200, Pakistan; \\ umairkhan@iba-suk.edu.pk \\ * Correspondence: zahir@ulm.edu.pk
}

Received: 28 August 2020; Accepted: 16 October 2020; Published: 19 October 2020

\begin{abstract}
In this research work, our goal is to scrutinize the case, where water-based nanofluids having single-wall and multi-wall carbon nanotubes (CNTs) flow through a vertical cone. The second law of thermodynamic is taken for the aim of scheming effective heat storage units. The body package is layered in convective heat and diluted permeable medium. The effects of Joule heating, rotary microorganisms, heat generation/absorption, chemical reactions, and heat radiation increase the novelty of the established model. By using a local similarity transformation technique, the partial differential equations (PDEs) change into a coupled differential equation. By using the numerical technique, bvp4c, to get the solution of the conservation equations and their relevant boundary conditions. The parameters appearing in the distribution analysis of the alliance are scrutinized in detail, and the consequences are depicted graphically. It can be perceived that in the situation of composed nanotubes, the velocity of fluid decreases as the magnetic field is increased.
\end{abstract}

Keywords: gyrotactic microorganisms; micropolar magnetohydrodynamics (MHD); Maxwell nanofluid; single wall carbon nanotubes (SWCNTs) and multi wall carbon nanotubes (MWCNTs); thermal radiation; chemical reaction; mixed convection; permeability

\section{Introduction}

In the present era, nanofluid is getting importance from the researchers due to its diverse application in the industrial field. For instance, they are available in polymer manufacturing, gas turbines, power generators, glass fabric, paper production, wire drawing, and many more. Nanofluid is a sort of heat transport medium containing nanoparticles under $100 \mathrm{~nm}$, which are reliably and consistently scattered in a base fluid like water, oil, and ethylene glycol. These scattered nanoparticles, for the most part, a metal or metal oxide massively improve the thermal conductivity of the nanofluid, upgrades conduction, and convection coefficients, mulling over more heat transport. Enhancing the thermal specifications of liquids leads to a greater level of connective flow in thermal units. For heat transfer enhancement, adding additives to the operant liquids for modifying their thermal features are very attractive method. For this, a way has been represented by enhancement in nanotechnology. The meaning of 'nanofluid' has been expressed by Choi [1] in 1995 for increasing heat transfer 
specifications of convectional fluids. A total report on the productivity of temperature transformation in the sunlight-based authority with the nanoliquid was done by Chen et al. [2]. They originate that the presentation of photo thermal change in the gatherer expanded by $96.93 \%$ and $52 \%$ at 30 and $75{ }^{\circ} \mathrm{C}$ separately, an entirely reasonable liquid for sun powered authorities which have the shortest fascination in low-temperatures. Oudina [3,4] examined nanomaterial conduct esoteric an annulus with different designs of temperature foundations. They used an arithmetical methodology and introduced soundness investigation. Mesoscopic line for investigating nanomaterial course through permeable area was introduced by Sheikholeslami [5]. They utilized Lorentz force to switch the stream style inside an opening. Chougule et al. [6] initiate that because of the low association of nano-powders, a pressure drop is critical in the curved cylinder when they utilized carbon nanotubes (CNTs) instead of unadulterated water. Besides, a curved cylinder with loop supplements improves better Nusselt number as a result of the rate of energy altercation increment because of this reality nano-powders have arbitrary and unpredictable movement in the liquids. Numerically, the plan assessment of a whirling stream microchannel for incredible warmth transition uses had been examined by Hartmann-Priesnitz et al. [7] who displayed the operant liquids as $\mathrm{Cu}-\mathrm{H}_{2} \mathrm{O}$ nanomaterial in the laminar stream. Ding et al. [8] portrayed nanofluids that depend on CNTs, which depend upon the heat of the base liquid, and set up that when the liquid heat is $25^{\circ} \mathrm{C}$, the warm conductivity can be expanded by up to $30 \%$, but can be increased by $79 \%$ observed at $40{ }^{\circ} \mathrm{C}$. Pop and Watanabe [9] carried out a theoretical analysis with the main aim to discuss the influence of injection/suction on fluid flowing over a cone with free convection and heat flux. The authors used different differential methods to solve the existing equation describing the flow. Xu [10] recently studied time-dependent hybrid nanofluid with mixed convection in rotating disks multiple kinds of nanoparticles are taken here. A numerical approach is used for the solution. Flaccid devices are used by investigators in an earlier investigation to improve the convective coefficient [11]. Many researchers used a combination of both concepts to enhance convective coefficient by use of insert with nanofluids. Heat transfer and friction element characteristics on warped tape with $\mathrm{Al}_{2} \mathrm{O}_{3} /$ water nanofluid are analyzed by Sharma et al. [12]. Zhang [13] has investigated adapted computational approaches in which a 2-D effective heat capacity model is used for forecasting the fleeting heat transmission procedure of the building envelopes equipped with phase change materials (PCM). The deviations against test data made by the principal procedures were viewed as especially bigger than the changed methodologies adjusted techniques. Sun et al. [14] have experimentally deliberated the heat transmission rate augmentation produced by natural convection of PCMs experiencing melting. The thermophysical properties of nanofluids have been discussed by Phuoc et al. [15]. The increase in the transfer of heat of SWCNTs-glycol-based nanofluids was examined by Harish et al. [16]. It was found that while $0.2 \%$ by volume of SNTs was added to ethylene glycol, the thermal conductivity increased by $14.8 \%$. The magnetohydrodynamic (MHD) 3-D Maxwell nanofluid boundary layer flow with convective boundary conditions on a biaxially stretched sheet was explored by Hayat et al. [17].

Nowadays the main concerns of scientists are to make the strategies that control the ingesting of skilled vitality. In the field of thermal structuring, the key objective is to achieve the best viability of contraptions and with the base loss of warmth, scouring, and spread during the mechanical procedures. All the heat gadgets take a shot at the guideline of thermodynamics and produce. Thermodynamic second laws used to look at the irreversibility in terms of the entropy age rate. Entropy growth is abused to elucidate the exhibition of various settings in present-day and structure solicitations. Entropy is imitative from the Greek word Entropia, which suggests "moving toward" or "alteration". Entropy figuring is basic as it orders the factors for energy forfeiture. Bejan [18] offered the clue of an entropy generation problem. Ellahi et al. [19] studied the influence of entropy optimization on natural convective nanoliquid stream. A multiple turbulator has been engaged by Sheikholeslami et al. [20] to enlarge the involvement of nanomaterial inside a tube. They verified that thermal irreversibility improves with the upsurge of subordinate flow. The related research work in a similar filed can be seen in [21,22]. 
The attractive properties of carbon nanotubes (CNTs) include mechanical and chemical stability, excellent thermal and electrical conductivity, lightweight and physicochemical reliability, making them a desirable material in the manufacture of electrochemical devices. Considering this exciting feature of carbon nanotubes, in this research work, our goal was to scrutinize the case where water-based nanofluids having single-wall and multi-wall CNTs flow through a vertical cone. The body package is layered in convective heat and diluted permeable medium. The effects of Joule heating, rotary microorganisms, and heat generation/absorption, chemical reactions, and heat radiation increase the novelty of the established model. By using a local similarity transformation technique, the PDE is changed into a coupled differential equation. By using the Homotopy analysis method to get the solution of the conservation equations and their relevant Boundary conditions. The parameters appearing in the distribution analysis of the alliance are scrutinized, and the consequences are depicted graphically. It can be perceived that on account of the two nanotubes, the velocity of fluid decreases as the magnetic is increased. Moreover, the thickness of moving microorganisms is decreased compared to more estimation of biological convection constants.

\section{Mathematical Analysis}

We suppose the flow of magnetohydrodynamic (MHD) mixed convective viscous water-based micropolar nanofluidic of CNTs on a vertical cone in a penetrable medium. The coordinate system for the flow phenomena is chosen is such a way that $\mathrm{x}$-coordinate is parallel with the direction of the fluid. Energy expression with thermal radiation dissipation, thermal flux, and Joule heating is measured. Irreversibility investigation with chemical reaction is investigated. Entropy rate is determined. Slip impact is likewise talked about. A magnetic field of constant strength $\beta_{0}$ is applied vertically. The temperature $(T)$ and concentration ponder $(C)$. Speeding up because gravity acts downwards. Problem geometry is featured in Figure 1.

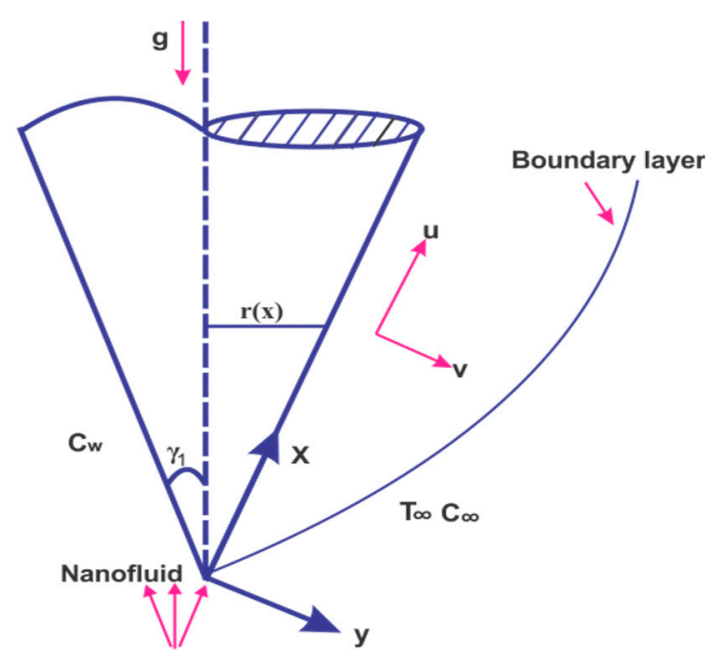

Figure 1. Schematic diagram for the flow direction.

From the above suppositions, the resulting modeled equations are:

$$
\begin{gathered}
(r u)_{x}+(r v)_{y}=0 \\
u u_{x}+v u_{x}+\lambda\left(u^{2} u_{x x}+v^{2} u_{y y}+2 u v u_{x y}\right)=\frac{\mu_{n f}}{\rho_{n f}} u_{y y}-\frac{\mu_{n f}}{\rho_{n f}} \frac{u}{K}+ \\
g\left[\beta\left(T-T_{\infty}\right)-\beta *\left(C-C_{\infty}\right)-\beta * \gamma\left(n-n_{\infty}\right)\right] \cos \gamma_{1}-\frac{\sigma_{n f} \beta_{0}^{2}}{\rho_{n f}} u+K N_{y} \\
u T_{x}+v T_{y}=\alpha_{n f} T_{y y}-\frac{1}{\left(\rho_{c p}\right)_{n f}}\left(q_{r}\right)_{y}+\frac{Q_{0}}{\left(\rho_{c p}\right)_{n f}}\left(T-T_{\infty}\right)+\frac{\sigma \beta_{0}^{2}}{\left(\rho_{c p}\right)_{n f}} u^{2}
\end{gathered}
$$




$$
\begin{gathered}
u N_{x}+v N_{y}=\frac{\gamma_{n f}^{*}}{\rho_{n f}} N_{y y}-\frac{k}{\rho_{n f}}\left(2 N+u_{y}\right) \\
u C_{x}+v C_{y}=D_{m} C_{y y}-k_{r}\left(C-C_{\infty}\right) \\
u n_{x}+v n_{y}+\frac{b W}{\left(C_{w}-C_{0}\right)}\left(n C_{y}\right)_{y}=D_{n} n_{y y}
\end{gathered}
$$

with the corresponding boundary conditions

$$
\begin{aligned}
& u=0, v=V_{1}, N=0, T_{y}=\frac{h_{f}\left(T_{f}-T\right)}{-k_{n f}}, \quad C_{w}=C=d x+C_{0}, n=n_{w}, \quad \text { At } y=a \\
& u \rightarrow 0, N \rightarrow 0, C \rightarrow C_{\infty}=C_{0}+e x, T \rightarrow T_{\infty}, n \rightarrow n_{w} \quad \text { At } y \rightarrow \infty
\end{aligned}
$$

where, $\left(\beta, \beta^{*}\right),\left(\mu_{n f}, \mu_{f}\right),\left(\rho_{C H T}, \rho_{f}\right), \beta_{0}, \alpha_{n f}, V_{0}, h_{f},(d, e), Q_{0},\left(\left(\rho_{c p}\right)_{f},\left(\rho_{c p}\right)_{n f}\right),\left(k_{f}, k_{n f}, k\right), D_{n}, k_{r}, W_{c}, D_{m}$, $q_{r}, \gamma_{1}$ indicate coefficients of thermal and solutal expansion, dynamic viscosities, densities, magnetic strength, thermal modified diffusivity, suction/injection parameter, convective parameter, dimensionless constants of concentration, heat generation/absorption parameter, heat capacities, thermal conductivity, diffusivity of microorganisms, chemical reaction rate, coefficient, extreme cell swimming motion, Brownian diffusion, radiation coefficient, and cone half-angle, respectively. In Equation (7) the term $V_{1}$ characterizes the mass transmission and defined as $V_{1}=-\left(\frac{3}{4} a x^{-1} R\left(a_{x}\right)^{\frac{1}{4}}\right) V_{0}$. In case of $V_{1}<0$, the mass transfer is for injection and $V_{1}>0$ shows suction.

$$
\begin{gathered}
\alpha_{n f}=\frac{k_{n f}}{\rho_{n f}\left(c_{p}\right)_{n f}}, \frac{k_{n f}}{k_{f}}=\frac{(1-\phi)+2 \Phi \frac{k_{C N T}}{k_{C N T}-k_{f}} \ln \left(\frac{{ }^{k_{C N T}+k_{f}}}{2 k_{f}}\right)}{(1-\phi)+2 \Phi \frac{k_{n f}}{k_{C N T}-k_{f}} \ln \left(\frac{{ }^{k_{C N T}+k_{f}}}{2 k_{f}}\right)} \\
v_{n f}=\frac{\mu_{n f}}{\rho_{n f}}, \gamma_{n f}^{*}=\left(\mu_{n f}+0.5 k\right) j, \rho_{n f}=(1-\phi) \rho_{f}+\phi \rho_{C N T}, \mu_{n f}=\mu(1-\phi)^{-2.5} \\
\frac{\sigma_{n f}}{\sigma_{f}}=1-\frac{3\left(\frac{\sigma_{s}}{\sigma_{f}}-1\right) \phi}{\left(\frac{\sigma_{s}}{\sigma_{f}}-1\right) \phi-\left(\frac{\sigma_{s}}{\sigma_{f}}+2\right)} \\
\psi=\alpha R a_{x}^{1 / 4} f(\eta), g(\eta)=\frac{C-C_{\infty}}{C_{w}-C_{0}}, \eta=\frac{y}{x} R a_{x}^{1 / 4} \\
\theta(\eta)=\frac{T-T_{\infty}}{T_{w}-T_{\infty}}, \mathrm{h}(\eta)=\frac{n-n_{\infty}}{n_{w}-n_{\infty}}, \mathrm{S}(\eta)=\frac{\rho_{f} x^{2} N R a_{x}^{-3 / 4}}{\mu_{f}}
\end{gathered}
$$

Using Equations (8) and (9), Equation (1) is satisfied and Equations (2)-(6) are written as

$$
\begin{gathered}
f^{\prime \prime \prime}+-k_{1} f^{\prime}+(1-\phi)^{2.5}\left(1-\phi+\phi \frac{\rho_{C N T}}{\rho_{f}}\right)\left[\theta-N_{r} g-R_{b} h\right]-(1-\phi)^{2.5} M f^{\prime}+ \\
K(1-\phi)^{2.5}\left(1-\phi+\phi \frac{\rho_{C N T}}{\rho_{f}}\right) S^{\prime}+\frac{1}{2 \operatorname{Pr}}(1-\phi)^{2.5}\left(1-\phi+\phi \frac{\rho_{C N T}}{\rho_{f}}\right) \\
\left\{f^{\prime 2}+\frac{1}{2} f f^{\prime \prime}\right\}-\frac{\beta}{4 \operatorname{Pr}}(1-\phi)^{2.5}\left(1-\phi+\phi \frac{\rho_{C N T}}{\rho_{f}}\right)\left(3 f^{\prime 3}+\frac{1}{4} f^{\prime \prime} f^{2}-\frac{5}{2} f^{\prime \prime} f^{\prime} f\right)=0 \\
\frac{k_{n f}}{k_{f}}(1+R d) \theta^{\prime \prime}+\frac{3}{4}\left(1-\phi+\phi \frac{\rho_{C N T}}{\rho_{f}}\right) f \theta^{\prime}+\gamma \theta+\operatorname{Pr} E c M f^{\prime 2}=0 \\
S^{\prime \prime}-\gamma *\left(2 S+\alpha f^{\prime \prime}\right)+\frac{1}{4 \operatorname{Pr}} \frac{(1-\phi)^{2.5}\left(1-\phi+\phi \frac{\rho_{C N T}}{\rho_{f}}\right)}{(1+0.5 K)}\left(5 S f^{\prime}+S^{\prime} f\right)=0 \\
g^{\prime \prime}+\frac{3}{4} S c f g^{\prime}-S c n f^{\prime}-G_{r} g=0 \\
h^{\prime \prime}+\frac{3}{4} L_{b} f h^{\prime}-P_{e}\left(h^{\prime} g^{\prime}+(h+\delta) g^{\prime \prime}\right)=0
\end{gathered}
$$




$$
\begin{aligned}
& f^{\prime}(0)=0, f(0)=V_{0}, \frac{k_{n f}}{k_{f}} \theta^{\prime}(0)=-B_{1}(1-\theta(0)), \mathrm{S}(0)=0, h(0)=1, g(0)=1-n, \\
& f^{\prime}(\infty) \rightarrow 0, S(\infty) \rightarrow 0, \theta(\infty) \rightarrow 0, h(\infty) \rightarrow 0, g(\infty) \rightarrow 0
\end{aligned}
$$

Non-dimensional form of parameters is specified and defined as below in Abbreviations.

\section{Entropy Generation Modeling}

To include the irreversibility sources, below equations can be used:

$$
\begin{aligned}
& S_{\text {gen }}^{\prime \prime \prime}=\frac{k_{n f}}{k_{f}}\left(1+\frac{16 T_{\infty}^{3} \sigma *}{3 k * k_{n f}}\right)\left(T_{y y}\right)+\frac{\mu_{n f}}{T_{\infty}}\left(u_{y}\right)^{2}+\frac{\sigma}{T_{\infty}} \beta_{0}^{2} u^{2}+\frac{\mu_{n f}}{T_{\infty} k} u^{2}+ \\
& \frac{R D}{C_{\infty}}\left(C_{y}\right)^{2}+\frac{R D}{T_{\infty}}\left(T_{y}\right)\left(C_{y}\right)
\end{aligned}
$$

where

$$
N_{G}=\frac{S^{\prime \prime \prime} g e n}{S^{\prime \prime \prime} 0}
$$

$\left(S^{\prime \prime \prime}\right.$ gen $)$ is irreversibility optimization rate and $\left(S^{\prime \prime \prime}\right)$ the characteristic irreversibility optimization rate signified by:

$$
\begin{aligned}
& N_{G}=\frac{k_{n f}}{k_{f}}(1+R) R a_{x} \theta^{\prime 2}+\frac{1}{(1-\phi)^{2.5}} \frac{B r R a_{x}}{\alpha}\left(f^{\prime \prime 2}+k_{1} f^{\prime 2}\right)+ \\
& \frac{B r R a_{x} M}{\alpha} f^{\prime 2}+\lambda\left(\frac{\xi}{\alpha}\right) R a_{x} g^{\prime 2}+\frac{\xi}{\alpha} R a_{x} \lambda \theta^{\prime} g^{\prime}
\end{aligned}
$$

where $\operatorname{Br}\left(=\frac{\mu_{f} U_{w}}{k_{f} \Delta T}\right)$ Brinkman number, $\alpha\left(=\frac{\Delta T}{T_{\infty}}\right)$ diffusion parameter, $\xi\left(=\frac{\Delta C}{C_{\infty}}\right)$ concentration ratio parameter, and $\lambda\left(=\frac{R D C_{\infty}}{k_{f}}\right)$ temperature difference parameter, respectively.

\section{Engineering Quantities}

4.1. Skin Friction Coefficients $C_{F x}$

Mathematically, it is defined as

$$
C_{F x}=\frac{2 \varphi_{w}}{\rho u_{\infty}^{2}}
$$

The dimensionless form is

$$
\operatorname{Re}_{x}^{1 / 2} C_{F x}=(1+K) \frac{1}{(1-\phi)^{2.5}} f^{\prime \prime}(0)
$$

In which $\operatorname{Re}_{x}^{1 / 2}$ designates Reynold number.

\subsection{Heat Transfer Rate}

$N u_{x}$ is

$$
N u_{x}=\frac{x Q_{w}}{k\left(\mathrm{~T}_{w}-\mathrm{T}_{\infty}\right)}
$$

where the heat flux $Q_{w}$ is

$$
\begin{aligned}
& Q_{w}=-\left.\frac{k_{n f}}{\kappa}\left(\frac{4 \sigma_{e}}{3 \kappa \kappa_{R}} \mathrm{~T}_{\infty}^{3}+1\right) \mathrm{T}_{y}\right|_{y=0} \\
& \operatorname{Re}_{x}^{-1 / 2} N u_{x}=-\frac{k_{n f}}{k_{f}}(1+R d) \theta^{\prime}(0)
\end{aligned}
$$




\subsection{Mass Transfer Rate}

Sherwood number $S h_{x}$ is defined as

$$
\begin{gathered}
S_{h x}=\frac{x h_{w}}{D_{B}\left(C_{w}-C_{\infty}\right)} \\
h_{w}=-\left.D_{B} C_{y}\right|_{y=0} \\
\operatorname{Re}_{x}^{-1 / 2} S h_{x}=-g^{\prime}(0)
\end{gathered}
$$

\subsection{Local Density of Motile Microorganisms}

Sherwood number $N_{n x}$ are stated as

$$
\begin{gathered}
N_{n x}=\frac{x Q_{n}}{D_{n}\left(n_{w}-n_{\infty}\right)} \\
Q_{n}=-\left.D_{n} n_{y}\right|_{y=0}
\end{gathered}
$$

After simplification

$$
\operatorname{Re}_{x}^{-1 / 2} N n_{x}=-h^{\prime}(0)
$$

\section{Solution Technique}

In this present work, the constitutive equations for the given problem are mathematically modeled under the following assumptions, heat generation/absorption, permeable medium, the geometry is taken to be a vertical cone, SWCNTs-water, and MWCNTs-water, convective boundary circumstances. The highly non-linear ODEs were obtained from PDEs by using the transformation technique. Hence, we employed a numerical system on the highly non-linear transformed differential equations.

Equations (12)-(16), along with boundary value problem (17) and (18), called a bvp4c just a name built-in Matlab function which is based on finite difference method and found the solutions computationally which is captured both in tables. Equations (1)-(6) and as well as in graphs. For this purpose, we can transform our differential equations into an arrangement of first order differential equations by letting the new factors

$$
f=y_{1}, f_{\prime}^{\prime}=y_{2}, f^{\prime \prime}=y_{3}, \theta=y_{4}, \theta \prime=y_{5}, S=y_{6}, S_{\prime}=y_{7}, g=y_{8}, g^{\prime}=y_{9}, h=y_{10}, h_{\prime}=y_{11}
$$

Exercising the above new variables in the Equations (12)-(16) then the following first order differential equations is achieved:

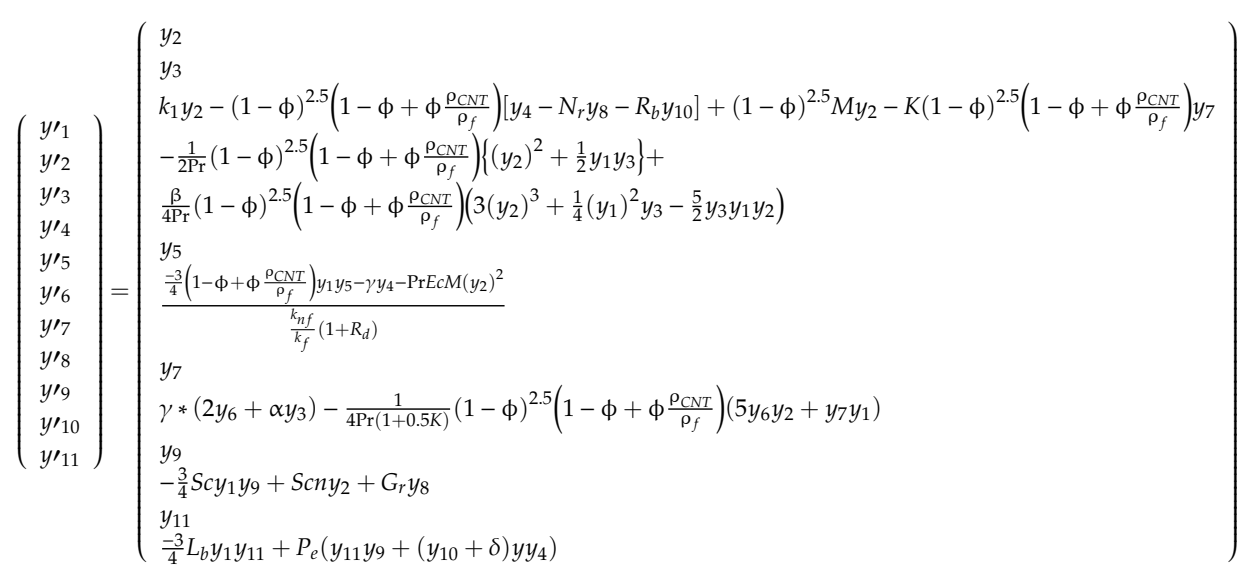


With initial conditions

$$
\left(\begin{array}{c}
y_{1}(0) \\
y_{2}(0) \\
y_{2}(\infty) \\
y_{5}(0) \\
y_{4}(\infty) \\
y_{6}(0) \\
y_{6}(\infty) \\
y_{8}(0) \\
y_{8}(\infty) \\
y_{10}(0) \\
y_{10}(\infty)
\end{array}\right)=\left(\begin{array}{l}
V_{0} \\
0 \\
0 \\
-\frac{k_{f}}{k_{n f}} B_{1}\left(1-y_{4}(0)\right) \\
0 \\
0 \\
0 \\
1-n \\
0 \\
1 \\
0
\end{array}\right)
$$

In this procedure, we can fix the pertinent parameters and then the solution starts with the initial guess supplied at the step size and changes the step size values to get the specified accuracy. The final number of the mesh length is attained by the function of Matlab called bvp4c throughout in the study to get the solutions. The region for the numerical solution should be finite, and the value is taken to be approximately 10 using $\eta=\eta_{\infty}$. Since, in the current problem we can find the solution for the SWCNTs-water and MWCNTs-water for a single guess of $\eta_{\infty}$. Here we have taken the value of $\eta_{\infty}=10$ for both SWCNT and MWCNT and found the profiles to reach the far field of the boundary layer conditions asymptotically.

\section{Validation of the Results}

Comparison between present and previous outcomes for justification is given in this section. Table 1 delineated to certify the accuracy of our current outcome with an available outcome in literature. The comparison of concentration gradient $S h_{x} \operatorname{Re}_{x}{ }^{-1 / 2}$ with variation of $S c$ while the rest of the parameters are associated with Ref. [21] and shown in Table 1. Obviously the outcome is in acceptable understanding.

Table 1. Numerical variation of mass transfer rate $S h_{x}$ and their comparison with Ref. [21] via various value of $S c$ at $G_{r}=n=0.1, N_{r}=0.5$.

\begin{tabular}{ccccc}
\hline \multirow{2}{*}{ Sc } & \multicolumn{2}{c}{ Ramzan et al. [21] } & \multicolumn{2}{c}{ Present Results } \\
\cline { 2 - 5 } & $\begin{array}{c}-\mathbf{g}^{\prime} \mathbf{( 0 )} \\
\text { SWCNT }\end{array}$ & $\begin{array}{c}-\mathbf{g}^{\prime} \mathbf{~ ( 0 )} \\
\mathbf{M W C N T}\end{array}$ & $\begin{array}{c}-\mathbf{g}^{\prime} \mathbf{( 0 )} \\
\text { SWCNT }\end{array}$ & $\begin{array}{c}-\mathbf{g}^{\prime} \mathbf{( 0 )} \\
\mathbf{M W C N T}\end{array}$ \\
\hline 0.1 & 0.31891 & 0.31882 & 0.3189450 & 0.3188567 \\
0.5 & 0.50221 & 0.50155 & 0.5022674 & 0.5015768 \\
0.9 & 0.74207 & 0.74087 & 0.7420467 & 0.7408564 \\
\hline
\end{tabular}

\section{Discussion}

This area is dedicated to the conversation and expectation of the effects of numerous parameters modeled from Equation (2) on $f^{\prime}(\eta)$. The impact of $M, \phi, N_{r}, R_{b}, k_{1}, \beta$ on velocity profile is discussed. Figure 2 presents the effect of the solid volume fraction $(\phi)$ of Maxwell micropolar nanofluid on velocity function. The motion of the nanoparticle increases for enlarging values of $(\phi)$. It is noted that $f^{\prime}(\eta)$ increases quickly for SWCNT in comparison to MWCNT. This augmentation in a motion of the micropolar nanoparticle is noted faster for single-wall carbon nanotube as compared to the multiwall carbon nanotube. Figure 3 presents the influence of $M$ on $f(\eta)$. The converse disparity is seen amongst $M$ and $f(\eta)$. The impact of the magnetic force is perpendicular to the Maxwell micropolar nanofluid flow direction executes augment to a resistive force. For a larger value of magnetic parameter $(M)$, the Lorentz forces enhance which raises the forces of resistance of the Maxwell micropolar motion which in turn reduces velocity $f^{\prime}(\eta)$. Figure 4 presents the impact of a suction parameter $V_{0}$ on $f^{\prime}(\eta)$. 
Here it is obvious from Figure 4, that enlarged estimation of the $V_{0}$ reduces the SWCNTs and MWCNTs Maxwell nanofluid motion. The impression of the buoyancy proportion parameter $N_{r}$ for SWCNTs and MWCNTs on $f(\eta)$ is presented in Figure 5. It is the ratio of nanofluid concentration and temperature difference amongst the layers as well as the intended operative resistance ratio at diverse values of $\beta$. From mathematical relation of $N_{r}$ it is clear that increasing concentration difference $\left(C_{W}-C_{0}\right)$ augmented $N_{r}$, while increasing temperature difference $\left(T_{f}-T_{0}\right)$ enhances $N_{r}$ Therefore, the augmented $N_{r}$ reduced the fluid motion. The impact of bio-convection Rayleigh number $R_{b}$ in Figure 6 as $R_{b}$ is a dimensionless number related to the buoyancy-driven of Maxwell micropolar nanofluid flow. From Figure 6 it is cleared that augmented value Rayleigh number $R_{b}$ reduced the Maxwell micropolar nanofluid motion. It is also found that velocity for MWCNT declines more quickly. In Figure 7, the result of the permeable parameter $k_{1}$ on $f(\eta)$ is drawn. As it is obvious that the permeable medium creates resistance to the fluid motion. From the figure it is perceived that $f^{\prime}(\eta)$ is decreases with higher permeability $k_{1}$. Additionally, the momentum boundary layer reduces with enhances value of $k_{1}$.

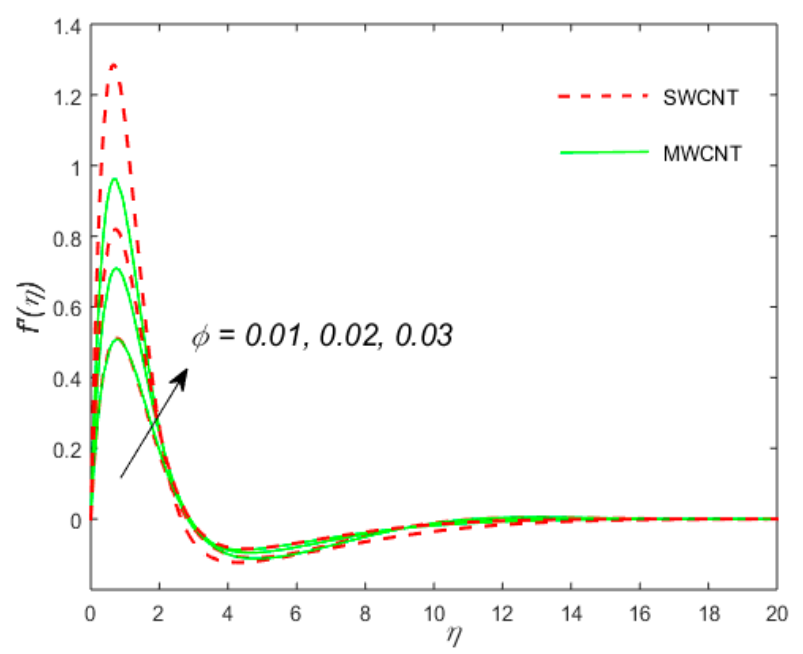

Figure 2. The variation of the velocity distribution profile $f^{\prime}(\eta)$ for the case of SWCNT and MWCNT versus the similarity variable for the distinct values of the nanoparticle volume fraction $(\phi)$.

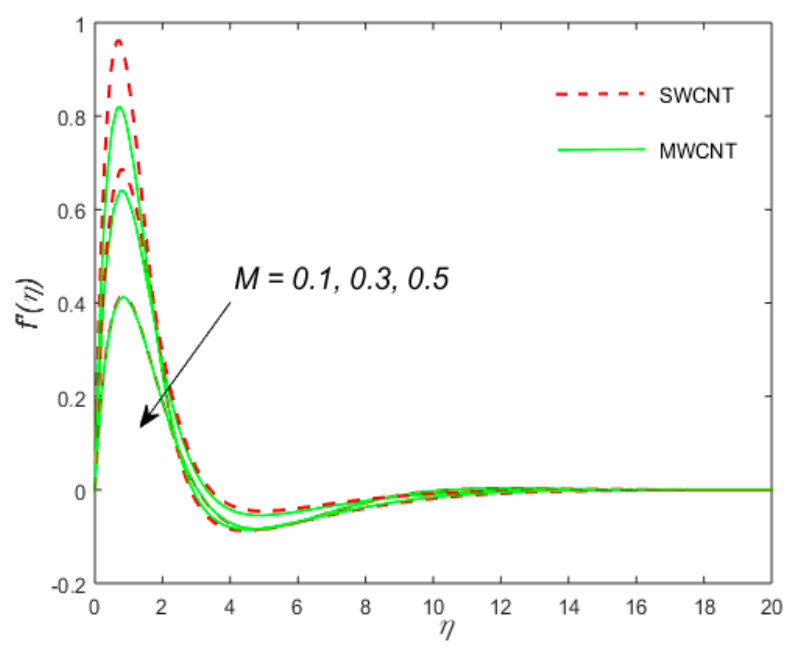

Figure 3. The variation of the velocity distribution profile $f^{\prime}(\eta)$ for the case of SWCNT and MWCNT versus the similarity variable for the distinct values of the magnetic parameter $(M)$. 


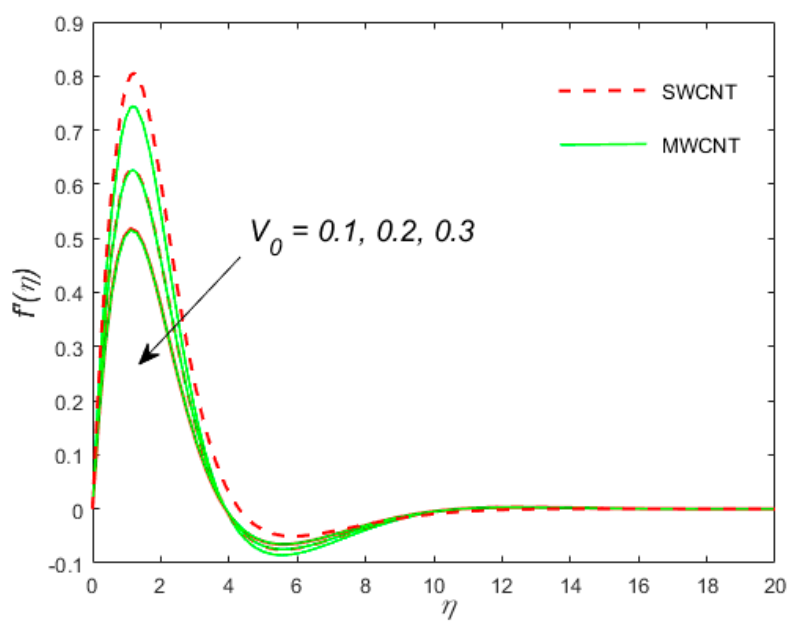

Figure 4. The variation of the velocity distribution profile $f^{\prime}(\eta)$ for the case of SWCNT and MWCNT versus the similarity variable for the distinct values of the suction parameter $\left(V_{0}\right)$.

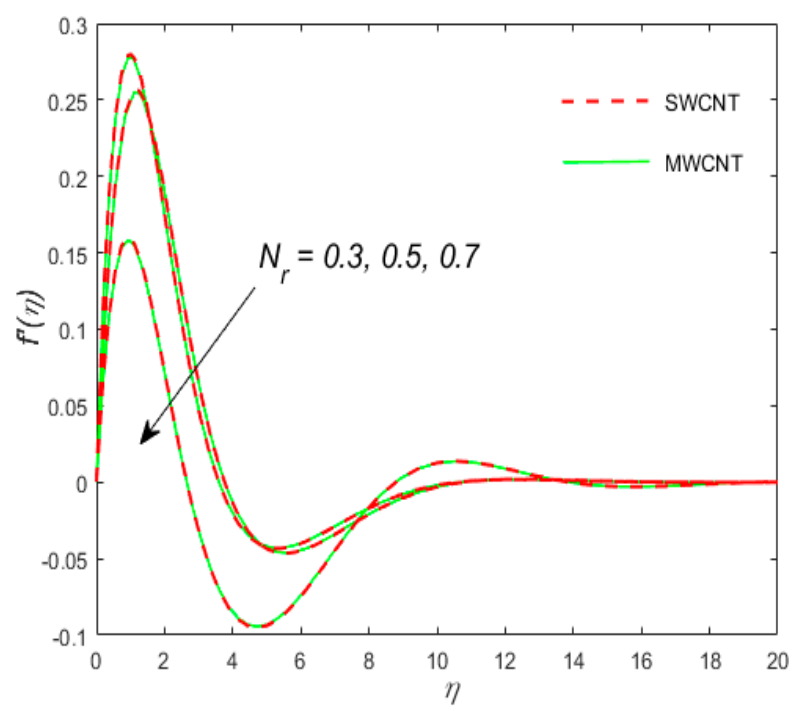

Figure 5. The variation of the velocity distribution profile $f^{\prime}(\eta)$ for the case of SWCNT and MWCNT versus the similarity variable for the distinct values of the buoyancy ratio parameter $\left(N_{r}\right)$.

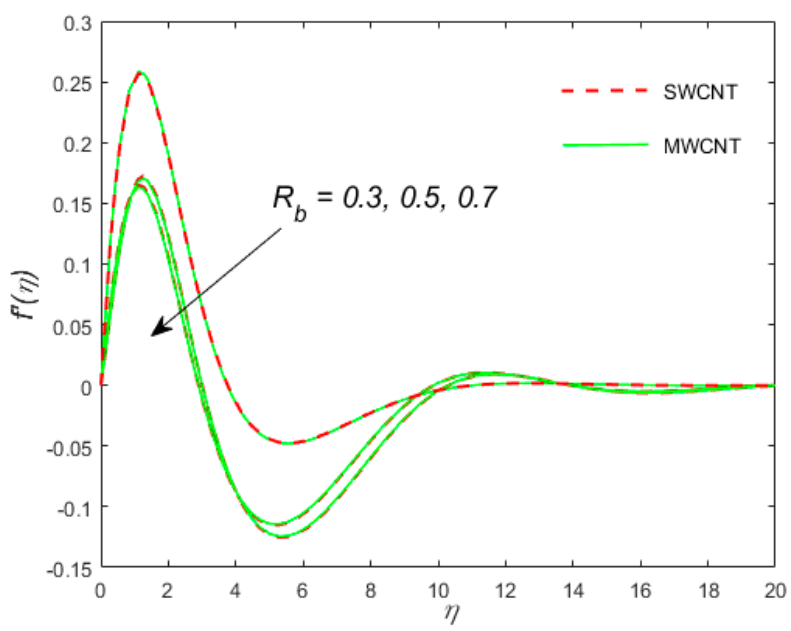

Figure 6. The variation of the velocity distribution profile $f^{\prime}(\eta)$ for the case of SWCNT and MWCNT versus the similarity variable for the distinct values of the bio-convection Rayleigh number $\left(R_{b}\right)$. 


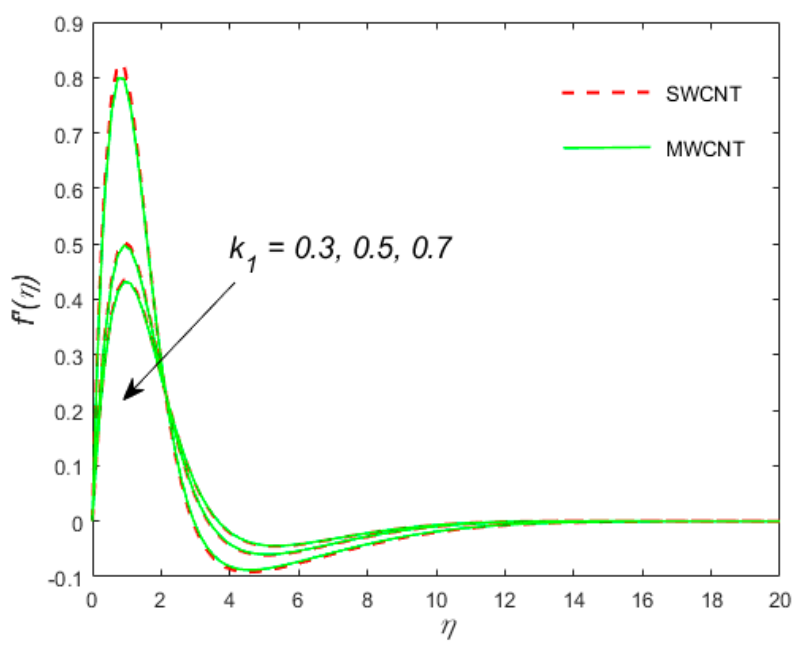

Figure 7. The variation of the velocity distribution profile $f^{\prime}(\eta)$ for the case of SWCNT and MWCNT versus the similarity variable for the distinct values of the porous parameter $\left(k_{1}\right)$.

\subsection{Temperature}

The substantial impacts of numerous factors displayed from temperature Equation (3) similar $\left(B_{1}\right)$, $(\mathrm{M}),\left(R_{d}\right)$, and $(E c)$ on temperature distribution profile are shown in Figures 8-11. The impression of Biot number $B_{1}$ on temperature distribution function $\theta(\eta)$ is presented in Figure 8. It is seen for the higher value of $B_{1}$ the temperature function $\theta(\eta)$ augmented for CNTs Maxwell micropolar nanofluid. Actually, increasing $B_{1}$ enhances the heat transmission from the surface becomes equivalent to that added from the exposed field which, in turn, conquers the temperature upsurge at the surface. Figure 9 labeled the impression of radiative parameter $R_{d}$ on the temperature distribution field $\theta(\eta)$ Augmentation in the $\theta(\eta)$ with enhancement radiation parameter $R_{d}$ is observed. Actually, intensification radiation causes additional heat which in turn escalates the CNTS Maxwell micropolar nanofluid temperature. Relation between Eckert number $E c$ and temperature distribution $\theta(\eta)$ is illustrated in Figure 10. Higher value of Ec amplified the kinetic energy of CNTs Maxwell micropolar nanofluid molecules which thus, enhanced the warmth transmission rate. Figure 11 shows the influence of magnetic induction $M$ on temperature distribution $\theta(\eta)$. For higher $(\mathrm{M})$ the strength of Lorentz forces become stronger which enhances the contrasting forces to the Maxwell micropolar nanofluid and results in the temperature distribution being $\theta(\eta)$ enhanced.

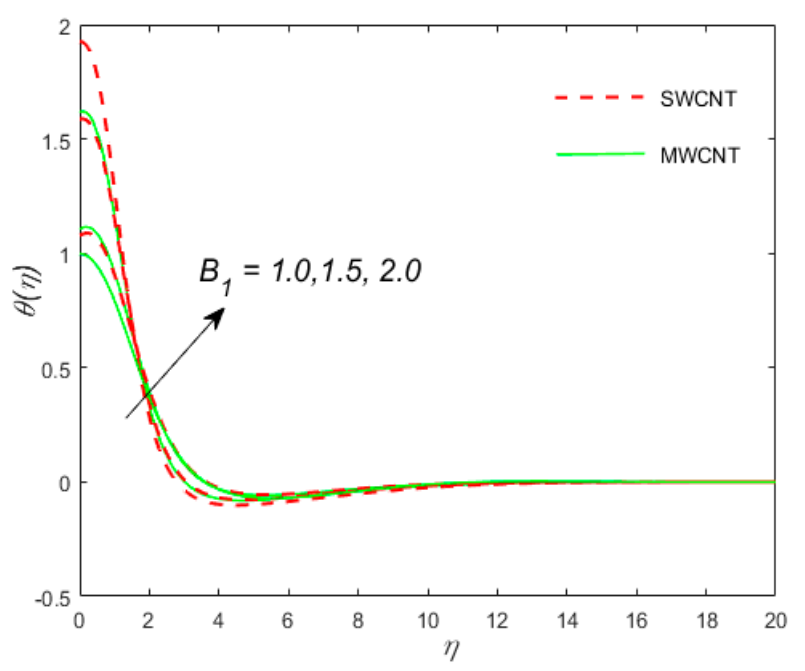

Figure 8. The impact of the temperature distribution profile $\theta(\eta)$ for the case of SWCNT and MWCNT versus the similarity variable for the distinct values of the Boit number $\left(B_{1}\right)$. 


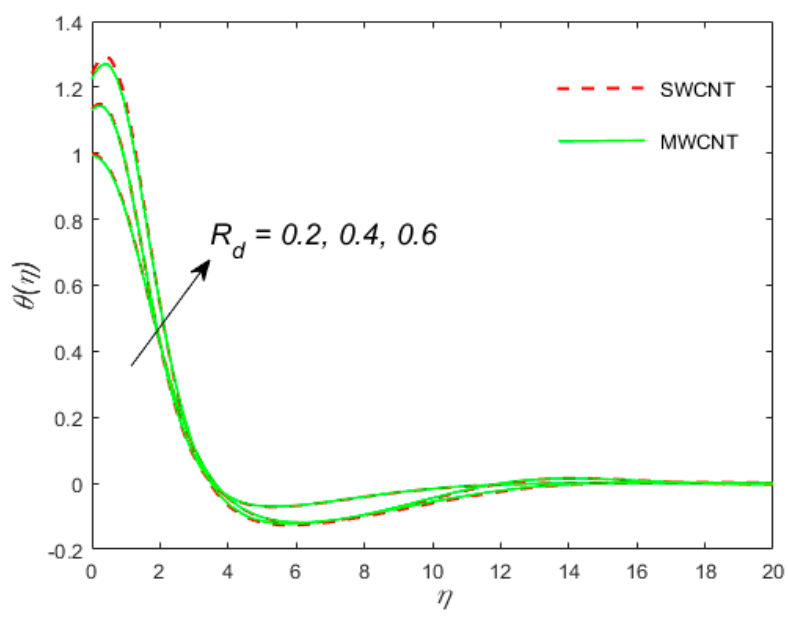

Figure 9. The impact of the temperature distribution profile $\theta(\eta)$ for the case of SWCNT and MWCNT versus the similarity variable for the distinct values of the radiation parameter $R_{d}$.

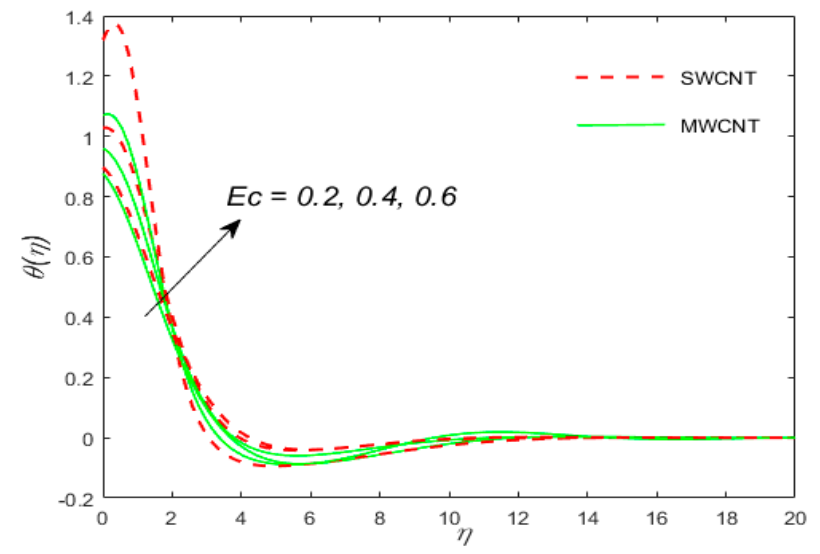

Figure 10. The impact of the temperature distribution profile $\theta(\eta)$ for the case of SWCNT and MWCNT versus the similarity variable for the distinct values of the Eckert number Ec.

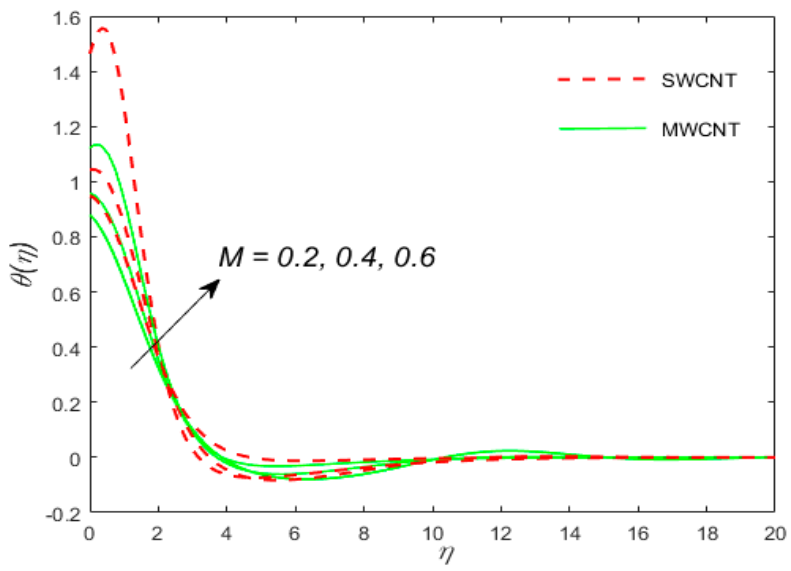

Figure 11. The impact of the temperature distribution profile $\theta(\eta)$ for the case of SWCNT and MWCNT versus the similarity variable for the distinct values of the magnetic parameter $M$.

\subsection{Micro Rotation Profile}

This region is stanch to the argument and anticipation of the effects of numerous parameters modeled from Equation (2) on $f(\eta)$. The impact of $K, \gamma^{*}$ and $\alpha$ on micro rotation velocity $S(\eta)$ is 
discussed, respectively. It is clear from the Figures $12-14$, that micro rotation velocity $S(\eta)$ augmented with higher value of $K, \gamma^{*}$, and $\alpha$.

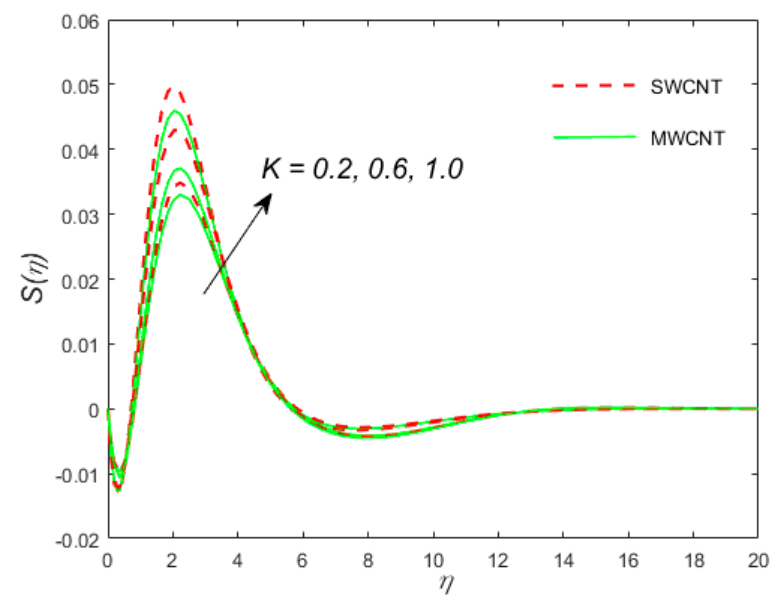

Figure 12. The variation of the micro rotation profile $S(\eta)$ for the case of SWCNT and MWCNT versus the similarity variable for the distinct values of the material parameter $K$.

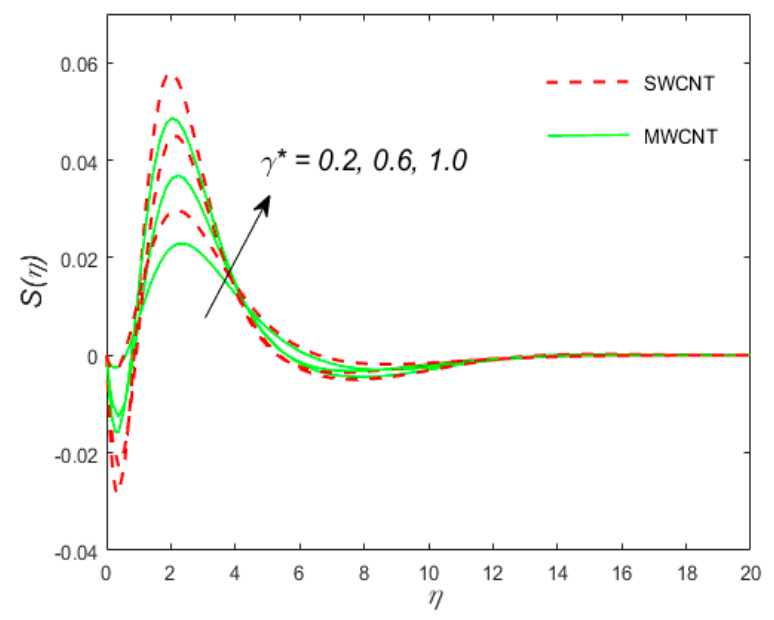

Figure 13. The variation of the micro rotation profile $S(\eta)$ for the case of SWCNT and MWCNT versus the similarity variable for the distinct values of the vortex velocity or material parameter $\gamma^{*}$.

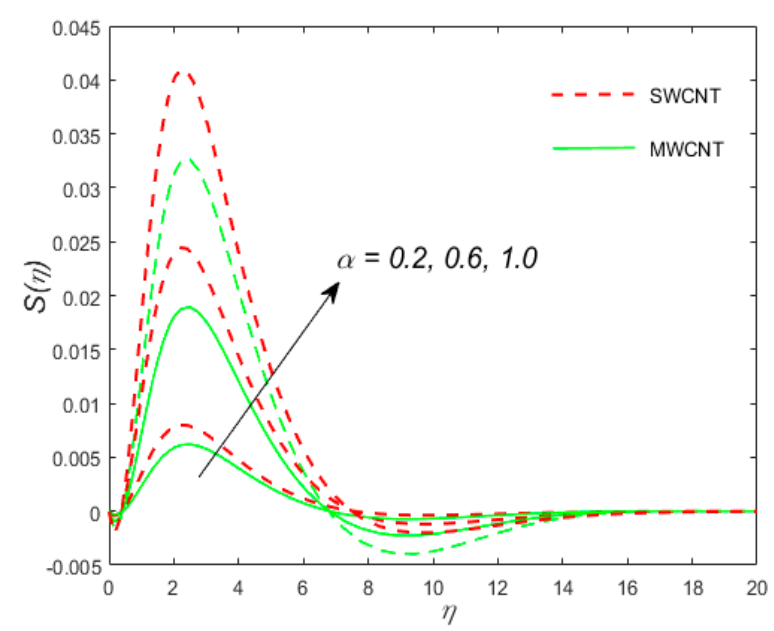

Figure 14. The variation of the micro rotation profile $S(\eta)$ for the case of SWCNT and MWCNT versus the similarity variable for the distinct values of the temperature difference parameter $\alpha$. 


\subsection{Concentration}

This part presented the discussion and anticipation of the effects of numerous parameters modeled from Equation (4) on $g(\eta)$. The impact of $S c$ and $G_{r}$ on concentration profile is discussed, respectively. Figure 15 is illustrated to examine the performance of $(S c)$ on $g(\eta)$. Higher values of $(S c)$ the diffusivity of mass declines and thus concentration is deteriorated. The influence of the chemical response factor $G_{r}$ on the concentration of Maxwell micropolar nanofluid is presented in Figure 16. It is observed that the augmented rate of $G_{r}$ reduces the concentration of Maxwell micropolar nanofluid.

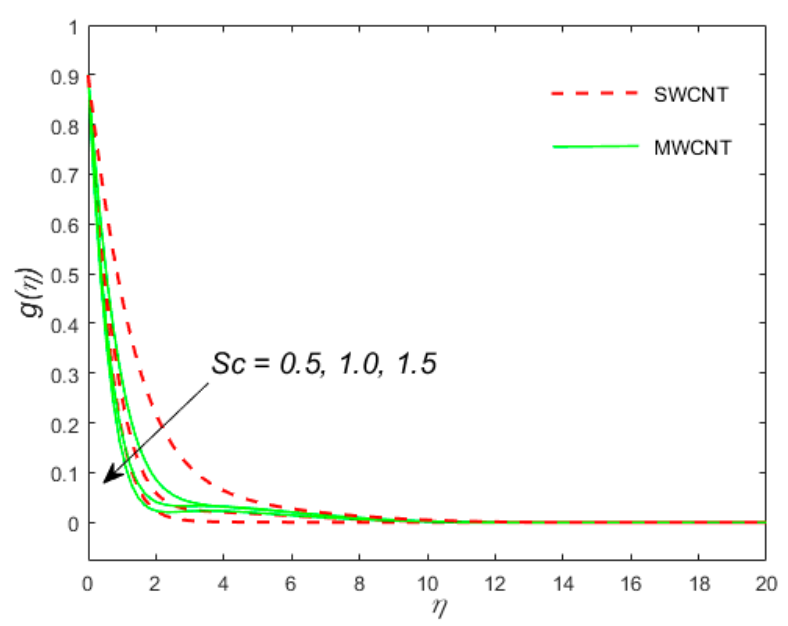

Figure 15. The variation of the concentration profile $g(\eta)$ for the case of SWCNT and MWCNT versus the similarity variable for the distinct values of the Schmidt number Sc.

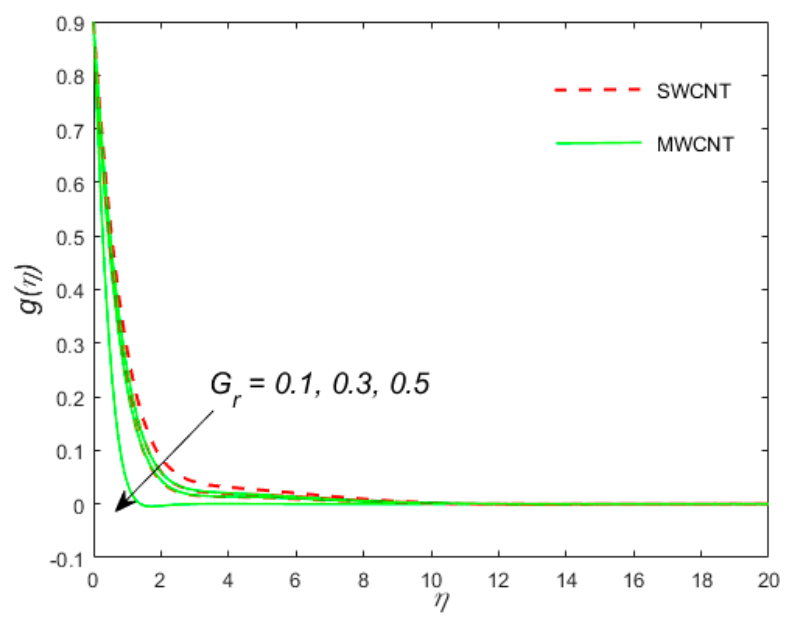

Figure 16. The variation of the concentration profile $g(\eta)$ for the case of SWCNT and MWCNT versus the similarity variable for the distinct values of the chemical response factor $G r$.

\subsection{Local Density of Motile Microorganisms}

The effects of $(\delta),(P e)$, and $\left(L_{b}\right)$ on Local density of motile microorganism's profile $h(\eta)$ appear in Figures 17-19. The impact of bio-convection constant $\delta$ on $h(\eta)$ is shown in Figure 17. The higher value of $\delta$ reduces $h(\eta)$ for both Maxwell micropolar nanotubes. The influences of $P e$ and $L_{b}$ are presented in Figures 18 and 19. Decreasing behavior are observed for both $L_{b}$ and $P e$. 


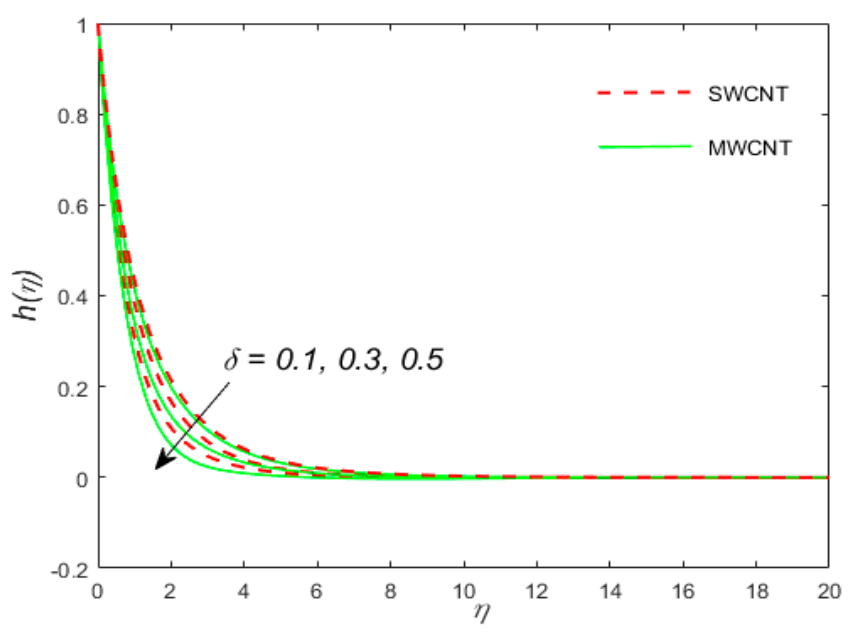

Figure 17. The variation of the local density of motile microorganisms $h(\eta)$ for the case of SWCNT and MWCNT versus the similarity variable for the distinct values of the bio-convection constant $\delta$.

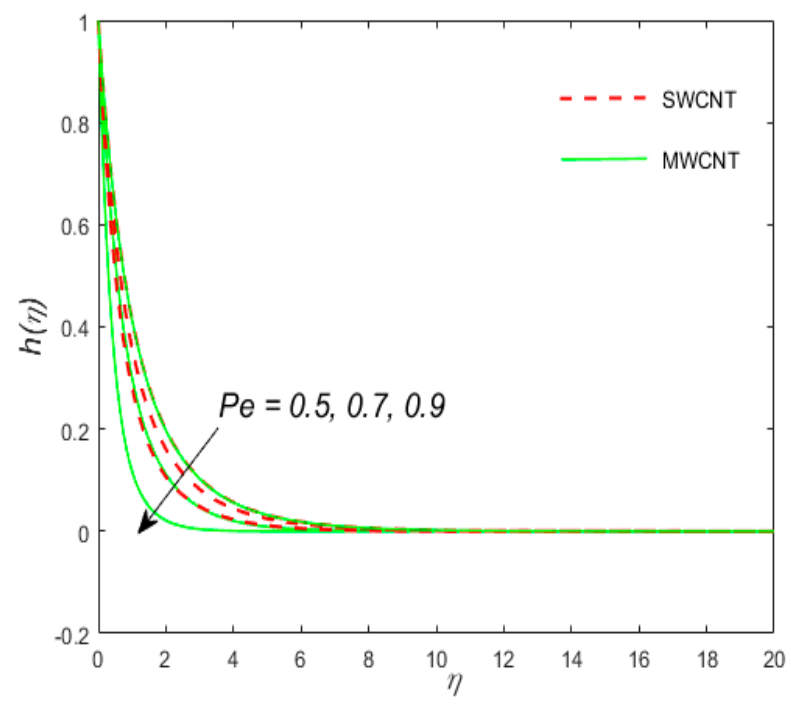

Figure 18. The variation of the local density of motile microorganisms $h(\eta)$ for the case of SWCNT and MWCNT versus the similarity variable for the distinct values of the bio-convection Peclet number $P e$.

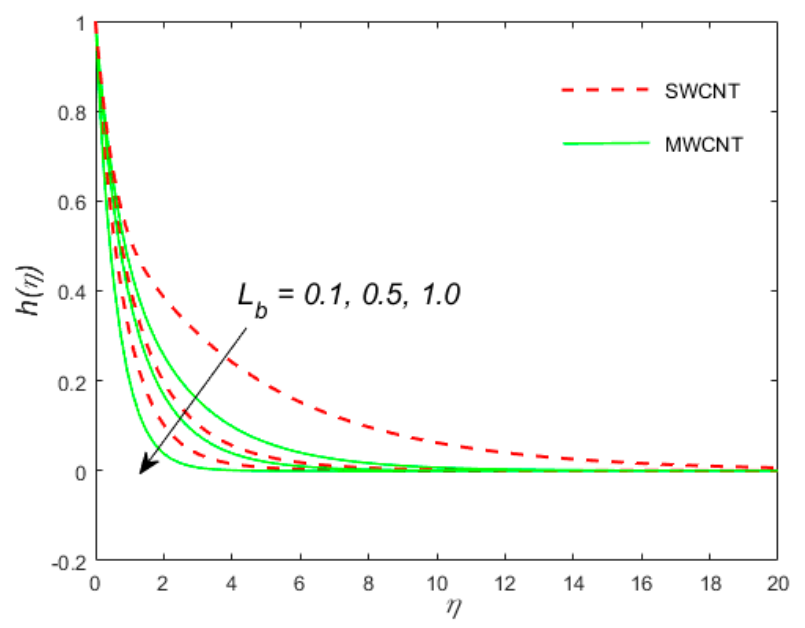

Figure 19. The variation of the local density of motile microorganisms $h(\eta)$ for the case of SWCNT and MWCNT versus the similarity variable for the distinct values of the bio-convection Lewis number $L_{b}$. 


\subsection{Entropy Optimization}

The meddled parameters $\xi, \lambda, \alpha, R a_{x}, K$, and $B r$ on $N G$ are exposed in Figures 20-25. Figures 20 and 21 illustrate the variation of $\xi$ and $\lambda$ on NG. The increasing value of concentration difference $\xi$ and diffusive constant $\lambda$ enhances the entropy NG of the nanofluid of SWCNTS and MWCNTS. The Significant effects of $\alpha$ on NG are illustrated in Figure 22. For a higher value of parameter $\alpha$ the entropy NG is found as decreasing function. Figures 23 and 24 show the impact of Reynold number $R a_{x}$ and micro rotation parameter $K$. Entropy optimization of CNTs nanofluid increases with increasing of $R a_{x}$ and $K$. In Figure 25 the impact of Brinkman number $B r$ is introduced. As a matter of fact, Brinkman number is a heat produced source inside the liquid moving district. The heat produced along with the heat moves from the divider and expands the entropy optimization.

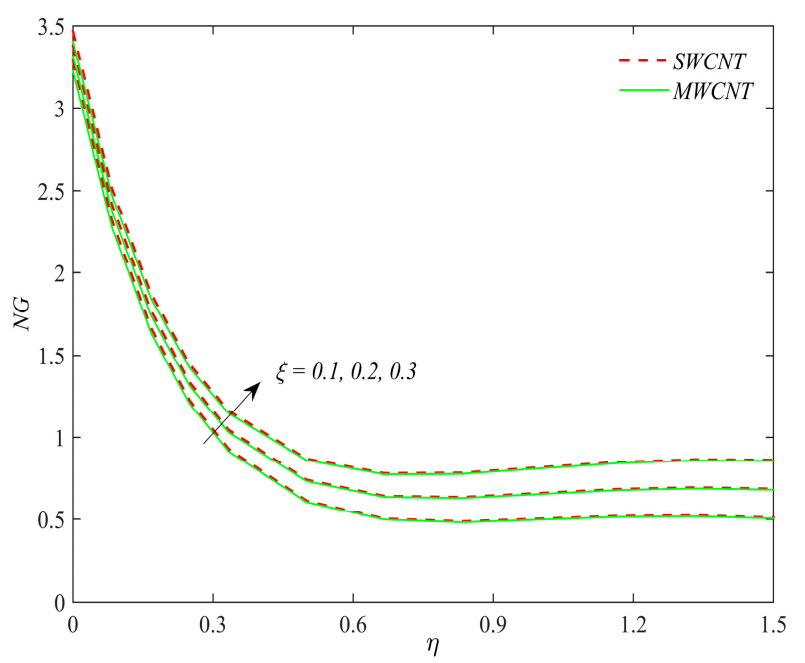

Figure 20. The variation of the entropy optimization NG for the case of SWCNT and MWCNT versus the similarity variable for the distinct values of the concentration difference parameter $\xi$.

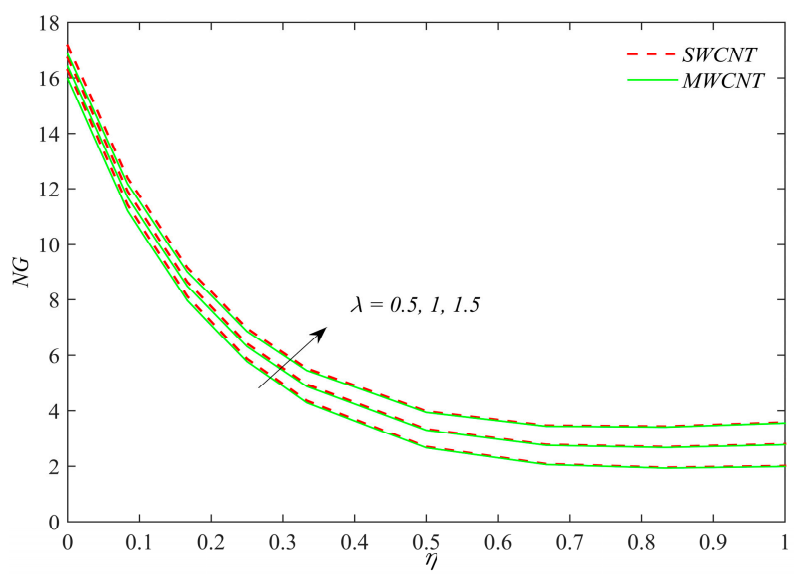

Figure 21. The variation of the entropy optimization NG for the case of SWCNT and MWCNT versus the similarity variable for the distinct values of the diffusive constant parameter $\lambda$. 


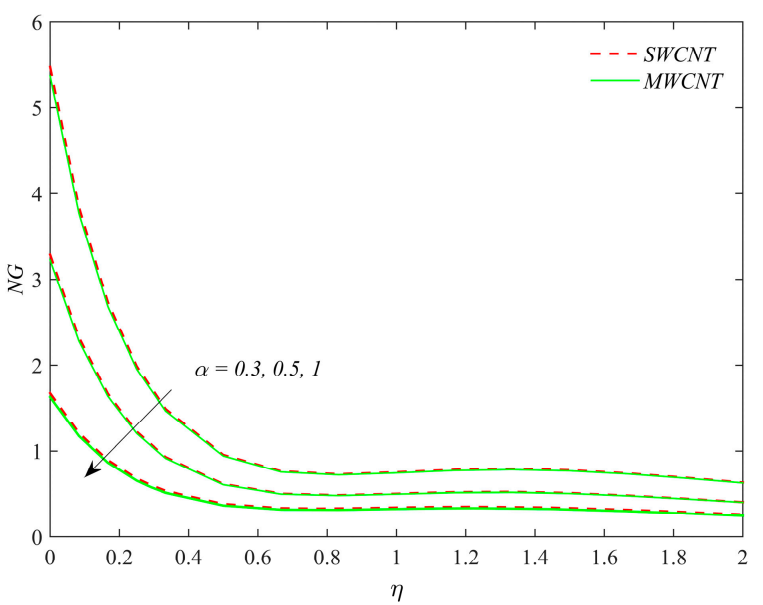

Figure 22. The variation of the entropy optimization NG for the case of SWCNT and MWCNT versus the similarity variable for the distinct values of the temperature difference parameter $\alpha$.

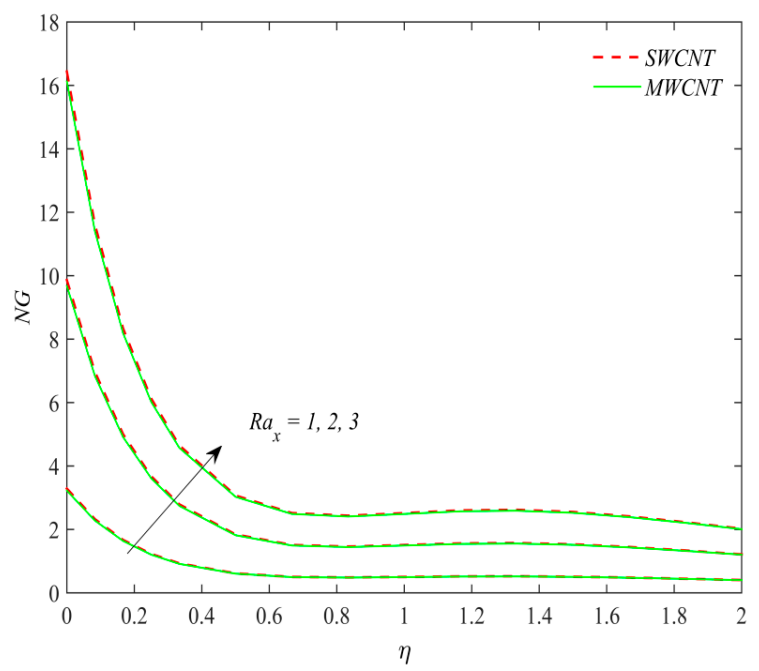

Figure 23. The variation of the entropy optimization NG for the case of SWCNT and MWCNT versus the similarity variable for the distinct values of the Reynold number $R a_{x}$.

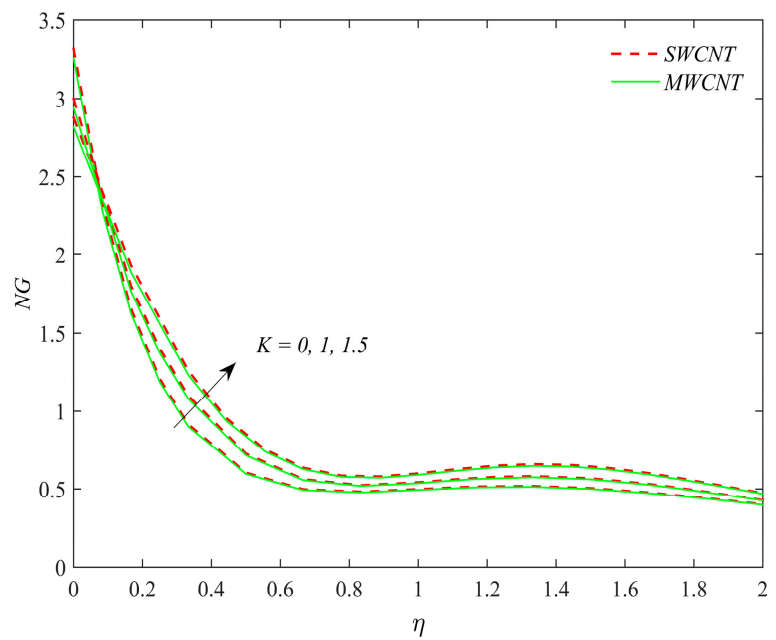

Figure 24. The variation of the entropy optimization NG for the case of SWCNT and MWCNT versus the similarity variable for the distinct values of the material parameter $K$. 


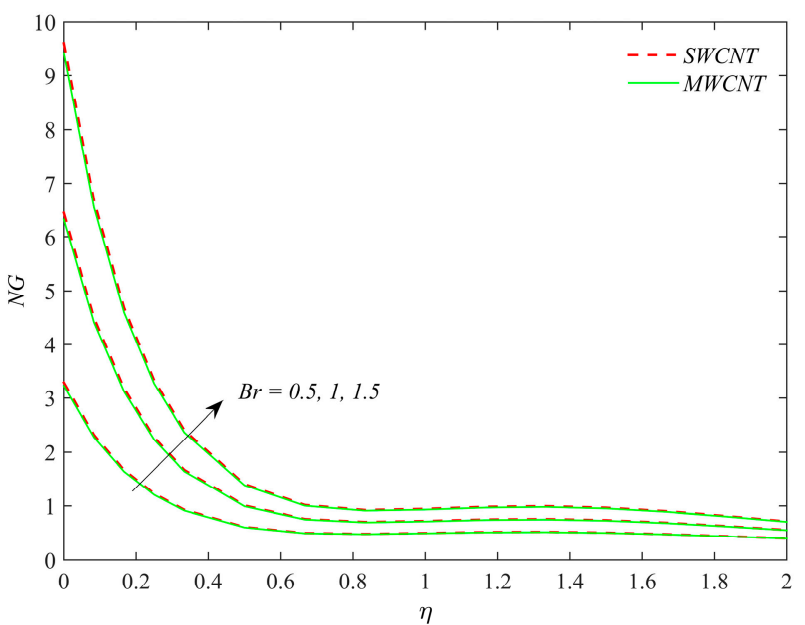

Figure 25. The variation of the entropy optimization NG for the case of SWCNT and MWCNT versus the similarity variable for the distinct values of the Brinkman number $\mathrm{Br}$.

\subsection{Engineering Quantities}

Performances of dissimilar engineering parameter on skin friction coefficient $C_{F x}$, temperature gradient $N u_{x}$, mass transfer $S h_{x}$, and local density of motile microorganisms $N_{n x}$ are presented in Tables 2-6. Various Thermal-physical properties of carbon nanotubes are shown in Table 2.

Table 2. Thermo-physical properties of base fluid and both type of carbon nanotubes (CNTs) i.e., SWCNTs and MWCNTs.

\begin{tabular}{cccc}
\hline Material & Water & SWCNT & MWCNT \\
\hline$C_{p}(\mathrm{j} / \mathrm{kgK})$ & 4179 & 425 & 796 \\
$\rho\left(\mathrm{kg} / \mathrm{m}^{3}\right)$ & 997.1 & 2600 & 1600 \\
$k(\mathrm{~W} / \mathrm{mK})$ & 0.613 & 6600 & 3000 \\
\hline
\end{tabular}

Table 3. Estimations of skin friction $f^{\prime \prime}(0)$ versus different evaluations of various parameters.

\begin{tabular}{|c|c|c|c|c|c|c|}
\hline \multirow{2}{*}{$\Phi$} & \multirow{2}{*}{$k_{1}$} & \multirow{2}{*}{$V_{0}$} & \multirow{2}{*}{$R_{b}$} & \multirow{2}{*}{$C$} & \multicolumn{2}{|c|}{$\frac{1}{(1-\phi)^{2.5}} f^{\prime \prime}(0)$} \\
\hline & & & & & SWCNTs & MWCNTs \\
\hline 0.01 & 0.5 & 1.0 & 0.1 & 0.1 & 1.8355 & 1.7511 \\
\hline 0.03 & - & - & - & - & 2.2996 & 1.8007 \\
\hline 0.05 & - & - & - & - & 2.4875 & 1.8673 \\
\hline- & 0.5 & - & - & - & 1.1596 & 1.1465 \\
\hline- & 0.7 & - & - & - & 1.4990 & 1.4987 \\
\hline- & 0.9 & - & - & - & 1.8355 & 1.8165 \\
\hline- & - & 0.5 & - & - & 2.8463 & 2.7389 \\
\hline - & - & 0.6 & - & - & 2.5630 & 2.5314 \\
\hline- & - & 0.7 & - & - & 2.3591 & 2.3284 \\
\hline - & - & - & 0.2 & - & 2.9477 & 2.2959 \\
\hline- & - & - & 0.3 & - & 1.9302 & 1.9281 \\
\hline- & - & - & 0.4 & - & 1.9201 & 1.8890 \\
\hline- & - & - & - & 0.1 & 2.1976 & 2.0633 \\
\hline- & - & - & - & 0.2 & 2.0917 & 2.0528 \\
\hline - & - & - & - & 0.3 & 2.0750 & 2.0450 \\
\hline
\end{tabular}


Table 4. Estimations of Nusselt number $-\frac{k_{n f}}{k}\left(1+R_{d}\right) \theta \prime(0)$ different evaluations of various parameters.

\begin{tabular}{ccccccc}
\hline $\boldsymbol{\Phi}$ & $\boldsymbol{R}_{\boldsymbol{d}}$ & $\boldsymbol{B}_{\mathbf{1}}$ & $\boldsymbol{M}$ & $\boldsymbol{E c}$ & \multicolumn{2}{c}{$-\frac{k_{n f}}{k}\left(1+\boldsymbol{R}_{\boldsymbol{d}}\right) \boldsymbol{\theta}^{\prime} \mathbf{( 0 )}$} \\
\cline { 6 - 7 } & & & & & SWCNTs & MWCNTs \\
\hline 0.01 & 0.1 & 1.0 & 0.1 & 0.5 & 0.0122 & 0.0138 \\
0.03 & - & - & - & - & 0.0200 & 0.0142 \\
0.05 & - & - & - & - & 0.0222 & 0.0153 \\
- & 0.2 & - & - & - & 0.0205 & 0.0323 \\
- & 0.3 & - & - & - & 0.0232 & 0.0181 \\
- & 0.4 & - & - & - & 0.0290 & 0.0160 \\
- & - & 0.5 & - & - & 0.0134 & 0.0133 \\
- & - & 0.7 & - & - & 0.0137 & 0.0138 \\
- & - & 1.0 & - & - & 0.0139 & 0.0177 \\
- & - & - & 0.1 & - & 0.0122 & 0.0119 \\
- & - & - & 0.2 & - & 0.0139 & 0.0138 \\
- & - & - & 0.3 & - & 0.0142 & 0.0141 \\
- & - & - & - & 0.1 & 0.0116 & 0.0115 \\
- & - & - & - & 0.5 & 0.0139 & 0.0138 \\
- & - & - & - & 1.0 & 0.0159 & 0.0158 \\
\hline
\end{tabular}

Table 5. Estimations of Sherwood number $-g_{\prime}(0)$ versus different evaluations of various parameters.

\begin{tabular}{cccccc}
\hline \multirow{2}{*}{$\boldsymbol{S c}$} & \multirow{2}{*}{$G_{r}$} & $\boldsymbol{n}$ & $N_{\boldsymbol{r}}$ & \multicolumn{3}{c}{$-g^{\prime}(\mathbf{0})$} \\
\cline { 5 - 6 } & & & & SWCNTs & MWCNTs \\
\hline 0.1 & 0.1 & 0.1 & 0.5 & 0.3430 & 0.3428 \\
0.5 & - & - & - & 0.6290 & 0.6102 \\
0.9 & - & - & - & 0.9227 & 0.8932 \\
- & 0.1 & - & - & 0.6290 & 0.6102 \\
- & 0.2 & - & - & 0.6977 & 0.6972 \\
- & 0.3 & - & - & 0.7513 & 0.7508 \\
- & - & 0.0 & - & 0.6290 & 0.6274 \\
- & - & 0.1 & - & 0.6147 & 0.6102 \\
- & - & 0.2 & - & 0.5782 & 0.6066 \\
- & - & - & 0.6 & 0.6072 & 0.6187 \\
- & - & - & 0.7 & 0.6005 & 0.6589 \\
- & - & - & 0.8 & 0.5954 & 0.6033 \\
\hline
\end{tabular}

Table 6. Values of Motile density number $-h \prime(0)$ versus various estimates of different parameters.

\begin{tabular}{|c|c|c|c|c|c|}
\hline \multirow{2}{*}{$L_{b}$} & \multirow{2}{*}{$P e$} & \multirow{2}{*}{$R_{b}$} & \multirow{2}{*}{$\delta$} & \multicolumn{2}{|c|}{$-h^{\prime}(0)$} \\
\hline & & & & SWCNTs & MWCNTs \\
\hline 0.5 & 0.5 & 0.1 & 0.1 & 0.7525 & 0.7515 \\
\hline 0.6 & - & - & - & 0.8386 & 0.8375 \\
\hline 0.7 & - & - & - & 0.9640 & 0.8806 \\
\hline- & 0.1 & - & - & 0.5504 & 0.5175 \\
\hline- & 0.2 & - & - & 0.5602 & 0.5760 \\
\hline- & 0.3 & - & - & 0.6074 & 0.6779 \\
\hline- & - & 0.0 & - & 0.7493 & 0.7483 \\
\hline- & - & 0.1 & - & 0.7441 & 0.7789 \\
\hline - & - & 0.2 & - & 0.7493 & 0.7847 \\
\hline- & - & - & 0.6 & 0.7792 & 0.7515 \\
\hline- & - & - & 0.7 & 0.7996 & 0.8050 \\
\hline- & - & - & 0.8 & 0.8494 & 0.8231 \\
\hline
\end{tabular}




\subsection{Surface Drag Force}

Table 3 show the numerical variation in term of skin friction $C_{F x}$. Numerical variation of $\Phi, k_{1}, V_{0}$, $R_{b}$, and $M$ are shown. It is observed that the higher value of $\Phi, k_{1}, V_{0}$, and $R_{b}$ augmented the surface drag force $C_{F x}$ for both SWCNTs and MWCNTs while the opposite impact is found for M.

\subsection{Heat Transfer Rate}

Table 4 show numerical variation in Nusselt number $N u_{x}$. Numerical variation of $\Phi, R_{d}, B_{1}, E c$, and $M$ are shown. It is observed that the higher value of $\Phi, R_{d}, B_{1}, E c$, and $M$ augmented the heat transfer rate for both SWCNTs and MWCNTs.

\subsection{Mass Transfer Rate}

Table 5 show the numerical variation in Sherwood number $S h_{x}$. Numerical variation of $S c, G_{r}, n$, and $N_{r}$ are shown. It is observed that the higher value of $S c, G_{r}, n$, and $N_{r}$ augmented the mass transfer rate $S h_{x}$ in both SWCNTs and MWCNTs while the opposite impact is found for $N_{r}$.

\subsection{Local Density of Motile Microorganisms $N_{n x}$}

Table 6 shows the numerical variation in local density of motile microorganisms $N_{n x}$. Numerical variation of $L_{b}, P e, R_{b}$, and $\delta$ are shown. It is observed that the higher value of $L_{b}, P e, R_{b}$, and $\delta$ augmented the local density of motile microorganisms both SWCNTs and MWCNTs while the opposite impact is found for $N_{r}$.

\section{Conclusions}

In this article we studied the flow of two vertical conical carbon nanotubes (SWCNT and MWCNT) under the action of aqueous nanofluids, accompanied by the movement of swirling microorganisms and the influence of solute stratification in porous media. The investigation is carried out in the presence of chemical reactions, heat generation/absorption, and Joule heating. The noticeable features of the modeled problem are:

- The motion of the nanoparticle increases for enlarging values of solid volume fraction $(\Phi)$.

- For a larger value of magnetic parameter $(M)$ the Lorentz forces enhance which raises the forces of resistance of the Maxwell micropolar motion which in turn reduces velocity $f(\eta)$.

- The augmented $N_{r}$ reduced the fluid motion.

- The momentum boundary layer reduces with enhances value of $k_{1}$.

- Micro rotation velocity $S(\eta)$ augmented with a higher value of $K, \gamma^{*}$, and $\alpha$.

- Augmentation in the $\theta(\eta)$ with enhancement radiation parameter $R_{d}$ is observed.

- The higher value of $E c$ amplified the kinetic energy of CNTs Maxwell micropolar nanofluid molecules, which thus enhanced the heat transmission rate.

- The augmented rate of $G_{r}$ reduces the concentration of Maxwell micropolar nanofluid.

- As the estimate of the number of Peclets increases, the number of motion densities also increases.

- With the rise in estimations of $P e, h(\eta)$ are increases.

- For both CNTs, $f(\eta)$ intensifies against rising values of suction. For rising values of $N_{r}, \theta(\eta)$ is reducing.

- For these CNTs, $g(\eta)$ is reduced on the increasing of $n$.

- For the growth estimates of $N_{r}, S h_{x}$ is reduced and raises against numerical values of $C_{r}$.

- For solid volume fraction $C_{f}$ is increased.

- Magnetic force $M$ reduces $N u_{x}$.

- A comparison between the present and previous outcomes for justification is given in Table 1. 
Author Contributions: Z.S. and E.A. modeled and solved the problem. Z.S. wrote the manuscript. M.J. and U.K. contributed in the numerical computations and plotting the graphical results. All the corresponding authors completed the manuscript after its internal evaluation. All authors have read and agreed to the published version of the manuscript.

Funding: This project was funded by the Deanship of Scientific Research (DSR), King Abdulaziz University, Jeddah, under grant No. (DF-143-130-1441).

Acknowledgments: The authors, therefore, gratefully acknowledge DSR for technical and financial support.

Conflicts of Interest: The authors declare that they have no conflict of interest.

\section{Abbreviations}

$\begin{array}{ll}\operatorname{Pr}=\frac{v_{f}}{\alpha} & \text { Prandtl number } \\ k_{1}=\frac{x^{2}}{K R a_{x}^{1 / 2}} & \text { Porous parameter } \\ M=\frac{\sigma \beta_{0}^{2} x^{2}}{\mu_{f} R a_{x}^{1 / 2}} & \text { Magnetic parameter } \\ S c=\frac{\alpha}{D_{m}} & \text { Schmidt number } \\ n=\frac{e}{d} & \text { Solutal stratification } \\ \gamma^{*}=\frac{k x^{2}}{\gamma_{n f}^{*} R a_{x}^{1 / 2}} & \text { Vortex velocity or material parameter } \\ \gamma=\frac{Q_{0} x^{2}}{\left(\rho c_{p}\right) R a_{x}^{1 / 2}} & \text { Heat suction/Injection parameter } \\ L_{b}=\frac{\alpha}{D_{n}} & \text { Bio-convection Lewis number } \\ R d=\frac{16 T_{\infty}^{3} \sigma}{3 k * k_{n f}} & \text { Radiation parameter } \\ \beta=\frac{\lambda_{1} \alpha R a_{x}^{1 / 2}}{x^{2}} & \text { Bio-convection Rayleigh number } \\ N_{r}=\frac{\beta *\left(C_{w}-C_{0}\right)}{\beta\left(T_{f}-T_{0}\right)} & \text { Buoyancy ratio parameter } \\ R_{b}=\frac{\beta * \gamma \Delta p \Delta n_{w}}{\beta\left(T_{f}-T_{0}\right)} & \text { Bio-convection Rayleigh number } \\ C_{r}=\frac{K_{r} x^{2}}{D_{m} R a_{x}^{1 / 2}} & \text { Chemical reaction parameter } \\ B_{1}=\frac{h_{f} x}{R a_{x}^{1 / 4} k_{f}} & \text { Boit number } \\ P e=\frac{b W}{D_{n}} & \text { Bio-convection Peclet number } \\ \delta=\frac{n_{\infty}}{n_{w}-n_{\infty}} & \text { Bio-convection constant } \\ \alpha=\frac{\Delta T}{T_{\infty}} & \text { Temperature difference parameter } \\ B r=\frac{\mu_{f} U_{w}}{k_{f} \Delta T} & \text { Brinkman number } \\ \xi=\frac{\Delta C}{C_{\infty}} & \text { concentration difference parameter, and } \\ \lambda=\frac{R D C_{\infty}}{k_{f}} & \text { diffusive constant parameter } \\ R a_{x} & \text { Reynold number }\end{array}$

\section{References}

1. Choi, S.U.; Eastman, J.A. Enhancing Thermal Conductivity of Fluids with Nanoparticles; Argonne National Lab: Lemonte, IL, USA, 1995.

2. Chen, L.; Liu, J.; Fang, X.; Zhang, Z. Reduced graphene oxide dispersed nanofluids with improved photo-thermal conversion performance for direct absorption solar collectors. Sol. Energy Mater. Sol. Cells 2017, 163, 125-133. [CrossRef]

3. Oudina, F.M. Convective heat transfer of Titania nanofluids of different base fluids in cylindrical annulus with discrete heat source. Heat Transf. Asian Res. 2019, 48, 135-147. [CrossRef]

4. Oudina, F.M. Numerical modeling of the hydrodynamic stability in vertical annulus with heat source of different lengths. Eng. Sci. Technol. Int. J. 2017, 20, 1324-1333.

5. Sheikholeslami, M.; Shah, Z.; Shafee, A.; Khan, I.; Tlili, T. Uniform magnetic force impact on water based nanofluid thermal behavior in a porous enclosure with ellipse shaped obstacle. Sci. Rep. 2019, 1196. [CrossRef]

6. Chougule, S.S.; Nirgude, V.V.; Gharge, P.D.; Mayank, M.; Sahu, S.K. Heat Transfer enhancements of low volume concentration CNT/water nanofluid and wire coil inserts in a circular tube. Energy Procedia 2016, 90, 552-558. [CrossRef] 
7. Herrmann-Priesnitz, B.; Calderón-Muñoz, V.R.; Valencia, A.; Soto, R. Thermal design exploration of a swirl flow microchannel heat sink for high heat flux applications based on numerical simulations. Appl. Therm. Eng. 2016, 109, 22-34. [CrossRef]

8. Ding, Y.; Alias, H.; Wen, D.; Williams, R.A. Heat transfer of aqueous suspensions of carbon nanotubes (CNT nanofluids). Int. J. Heat Mass Transf. 2006, 49, 240-250. [CrossRef]

9. Pop, I.; Watanabe, T. Free convection with uniform suction or injection from a vertical cone for constant wall heat flux. Int. Commun. Heat Mass Transf. 1992, 19, 275-283. [CrossRef]

10. $\mathrm{Xu}, \mathrm{H}$. Modelling unsteady mixed convection of a nanofluid suspended with multiple kinds of nanoparticles between two rotating disks by generalized hybrid model. Int. Comm. Heat Mass Tran. 2019, 108, 104275. [CrossRef]

11. Rohsenow, W.M.; Hartnett, J.P.; Cho, Y.I. Handbook of Heat Transfer; McGraw-Hill Book Co.: New York, NY, USA, 1985. [CrossRef]

12. Sharma, K.V.; Sundar, L.S.; Sarma, P.K. Estimation of heat transfer coefficient and friction factor in the transition flow with low volume concentration of $\mathrm{Al}_{2} \mathrm{O}_{3}$ nano fluid flowing in a circular tube and with wisted tape insert. Int. Commun. Heat Mass Transf. 2009, 36, 503-507. [CrossRef]

13. Zhang, Y. Modified computational methods using effective heat capacity model for the thermal evaluation of PCM outfitted walls. Int. Commun. Heat Mass Transf. 2019, 108, 104278. [CrossRef]

14. Sun, X.; Zhang, Q.; Medina, M.A.; Lee, K.O. Experimental observations on the heat transfer enhancement caused by natural convection during melting of solid-liquid 17 phase change materials (PCMs). Appl. Energy 2016, 162, 1453-1461. [CrossRef]

15. Phuoc, T.X.; Massoudi, M.; Chen, R.H. Viscosity and thermal conductivity of nanofluids containing carbon nanotubes stabilized by Chitosan. Int. J. Therm. Sci. 2011, 50, 12-18. [CrossRef]

16. Harish, S.; Ishikawa, K.; Einarsson, E.; Aikawa, S.; Chiashi, S.; Shiomi, J.; Maruyama, S. Enhanced thermal conductivity of ethylene glycol with single-walled carbon nanotube inclusions. Int. J. Heat Mass Transf. 2012, 55, 3885-3890. [CrossRef]

17. Hayat, T.; Muhammad, T.; Shehzad, S.A.; Chen, G.Q.; Abbas, I.A. Interaction of magnetic field in flow of Maxwell nanofluid with convective effect. J. Magn. Magn. Mater. 2015, 389, 48-55. [CrossRef]

18. Bejan, A. A study of entropy generation in fundamentsl convective heat transfer. J. Heat Trans. 1979, 101, 718-725. [CrossRef]

19. Ellahi, R.; Hassan, M.; Zeeshan, A.; Khan, A.A. The shape effects of nanoparticles suspended in HFE-7100 over wedge with entropy generation and mixed convection. Appl. Nanosci. 2016, 6, 641-651. [CrossRef]

20. Sheikholeslami, M.; Jafaryar, M.; Hedayat, M.; Shafee, A.; Li, Z.; Khang Nguyen, T.; Bakouri, M. Heat transfer and turbulent simulation of nanomaterial due to compound turbulator including irreversibility analysis. Int. J. Heat Mass Transf. 2019, 137, 1290-1300. [CrossRef]

21. Ramzan, M.; Mohammad, M.; Howari, F. Magnetized suspended carbon nanotubes based nanofluid flow with bio-convection and entropy generation past a vertical cone. Sci. Rep. 2019, 9, 12225. [CrossRef] [PubMed]

22. Sarafraz, M.M.; Reza Safaei, M.; Goodarzi, M.; Arjomandi, M. Reforming of methanol with steam in a micro-reactor with $\mathrm{Cu}-\mathrm{SiO}_{2}$ porous catalyst. Int. J. Hydrogen Energy 2019, 44, 19628-19639. [CrossRef]

Publisher's Note: MDPI stays neutral with regard to jurisdictional claims in published maps and institutional affiliations.

(C) 2020 by the authors. Licensee MDPI, Basel, Switzerland. This article is an open access article distributed under the terms and conditions of the Creative Commons Attribution (CC BY) license (http://creativecommons.org/licenses/by/4.0/). 